\title{
Editorial: Ten recommendations for enhancing urban design teaching and learning
}

\author{
Roy Strickland \\ Professor of Architecture, Taubman College of Architecture and Urban \\ Planning, University of Michigan, Ann Arbor, MI, USA; International \\ Visiting Professor of Urban Design, South China University of Technology, \\ Guangzhou, China
}

The following papers comprise a themed issue of the journal of Urban Design and Planning focused on urban design education. Written by faculty from Delft University of Technology, Harvard University, Tsinghua University, the University of Cincinnati, the University of Michigan and the University of Pennsylvania, they provide a concise, international overview of current urban design pedagogy and its design and research outcomes in China, the Netherlands, South Korea and the USA. The papers reflect the perspectives of a long-established urban design programme (Delft) and one relatively new (Tsinghua), and address the roles of history (Cincinnati and Michigan) and landscape architecture (Harvard) in urban design education. By discussing issues such as programme and curriculum development, design-based education and research, and inter-disciplinary study, they offer insights into the opportunities and challenges facing urban design education in a world where, for the first time, more than half of people live in cities.

This essay supplements the papers by presenting ten recommendations to graduate-level urban design programmes toward fostering a culture of learning that will deepen students' knowledge and appreciation of urban design. Formulated by an urban design teacher and programme director, the recommendations address matters of admissions, curriculum, and studio and course structure and are posed below as responses to real-world conditions that students will find on graduation.

1) With the majority of the world's population living in cities, urban design is a global field.

Response: To help prepare students for global practice, urban design programmes should admit students from around the world. By doing so they will offer exposure to international urban conditions and cultures through that most powerful educational tool, the teaching and learning that occurs between students. By calling on students' backgrounds and experiences in design and research projects such teaching and learning can also be formalised and made integral to studios and courses. (Figure 1)

2) Urban design is inter-disciplinary, occupying the crossing of architecture, urban planning, landscape architecture and related fields such as civil engineering, environmental conservation, real estate development and historic preservation.

Response: Rather than limit themselves to students with professional degrees in architecture, as is often the case in graduate-level programmes, urban design programmes should enroll students from fields such as the ones noted above. Through student-to-student teaching and learning rising urban designers will broaden their understanding of and approaches to the field.

3) Given that urban design is inter-disciplinary and global, effective communication is essential to practice.

Response: Clear, jargon-free language should be the hallmark of urban design programmes. It is fundamental in order to break down divisions between students from different national and disciplinary backgrounds and is essential for communication between urban designers and the world in which they practice where stakeholders and decision-makers are unversed in professional jargon.

4) Urban design is not just design but requires analytical and research skills and historical and cultural awareness.

Response: To the degree possible, urban design programmes should integrate their studios and courses to discourage intellectual and disciplinary silos. Fold history, theory, economics, sociology and ecology, among others, into studio projects even as students may have separate courses in these subjects. Meanwhile, fold quick urban design exercises into subject courses to apply the courses' lessons directly to urban design. (Figure 2)

5) As can be seen in the range of urban design practices and projects around the world, there is no single way to practice urban design.

Response: As students are grounded in their programme's academic requirements, they should also be encouraged to pursue existing and new areas of interest to help them develop their own approaches to design. Studio projects can include periods when students assume expert status based on their disciplinary backgrounds, sharing knowledge and developing new knowledge with their colleagues, the blend of existing and new knowledge leading to a variety of approaches to design. In academic 
Editorial: Ten recommendations for enhancing urban design teaching and learning

Strickland

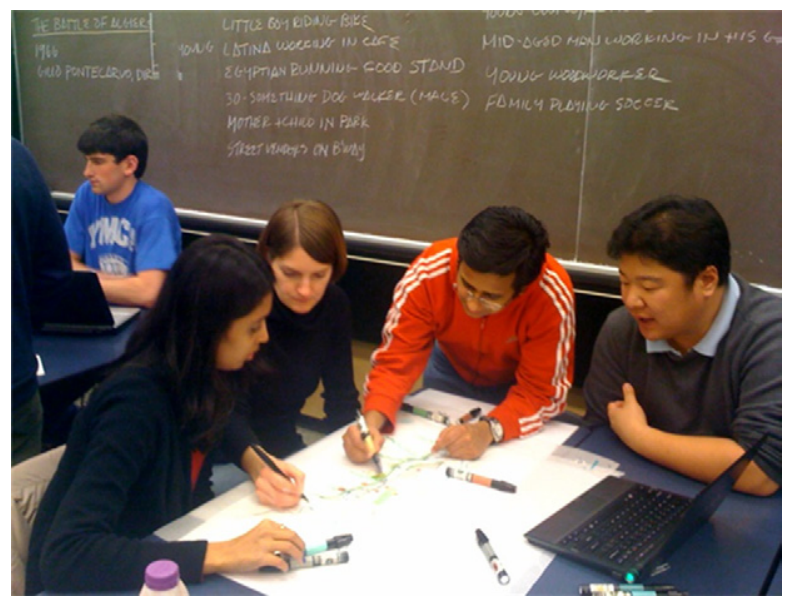

Figure 1. An international, interdisciplinary team at work on an urban design studio project

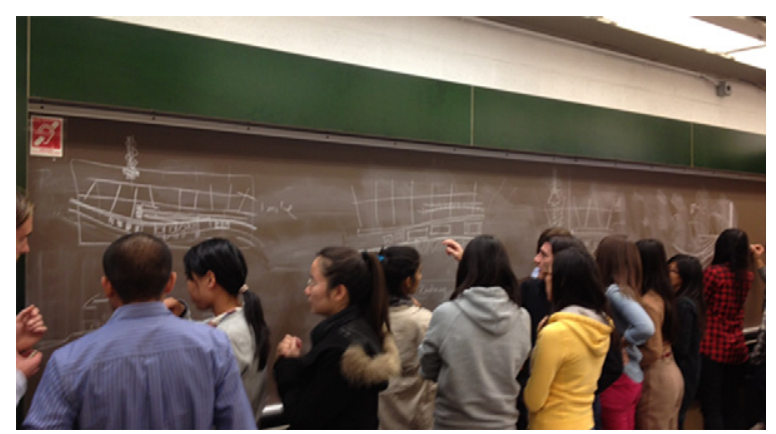

Figure 2. Break out session during an urban design methods and theory seminar. One of several during the semester, it promotes direct application of seminar's lessons to design

courses, research components can enable students to pursue questions of interest, reinforcing the development of their approaches to urban design.

6) Due to the scale and complexity of projects, urban design is not a singular (individualistic) pursuit.

Response: Promote teamwork in design and research projects at all scales. In both studios and related courses, rotate students between teams to assure their exposure to multiple views and backgrounds.

7) Usually the decision-makers for urban design projects are not other urban designers but stakeholders who include the public, politicians, developers and government agencies, among others.

Response: During studio pin-ups and reviews, have students role-play a variety of stakeholders. In assuming alternative identities they will see and criticise projects from perspectives other than urban design (with the added benefit of practising jargon-free communication).
8) Urban design is a public profession; its outcomes affect nations, regions, cities and neighbourhoods.

Response: Encourage studio and research projects in public that require students to communicate and represent their work outside of the academic setting. To the degree possible, identify projects with real stakeholders who can help set project goals and participate in project design development. Such projects need not dominate curriculum but can be mixed with academic projects. (Figure 3)

9) Given the size and complexity of urban design projects and the changing forces and actors involved in bringing them to fruition, urban design is often a non-linear process.

Response: Rather than arrange studio projects as a lockstep process moving from analysis to programme, concept, design development, design resolution and representation, make the process fluid so that cross-disciplinary teamwork and self-identified areas of exploration enable students to move back and forth in the design process. With proper pacing, studio projects thus arranged enable analysis and design discoveries to occur even late in the design process, enriching projects and enhancing their educational value. (Figure 4)

10) Real urban design projects can take years, even decades to come to fruition.

Response: Make design guidelines part of studio projects and the subject of research and discussion in other courses. Guidelines serve as the bridge between urban designers' visions and projects' ultimate outcomes. Indeed, they can be among the most important and influential parts of projects as different actors over time participate in project delivery, making them critical to urban design education. (Figure 5)

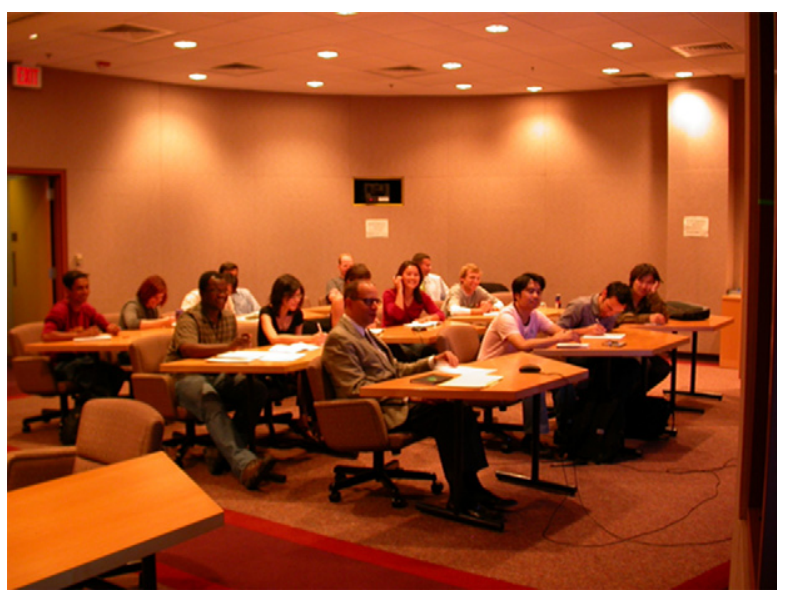

Figure 3. Projects in public. Members of urban design studio communicating via video conferencing with off-campus project stakeholders 
Urban Design and Planning Volume 170 Issue DP3
Editorial: Ten recommendations for enhancing urban design teaching and learning

Strickland

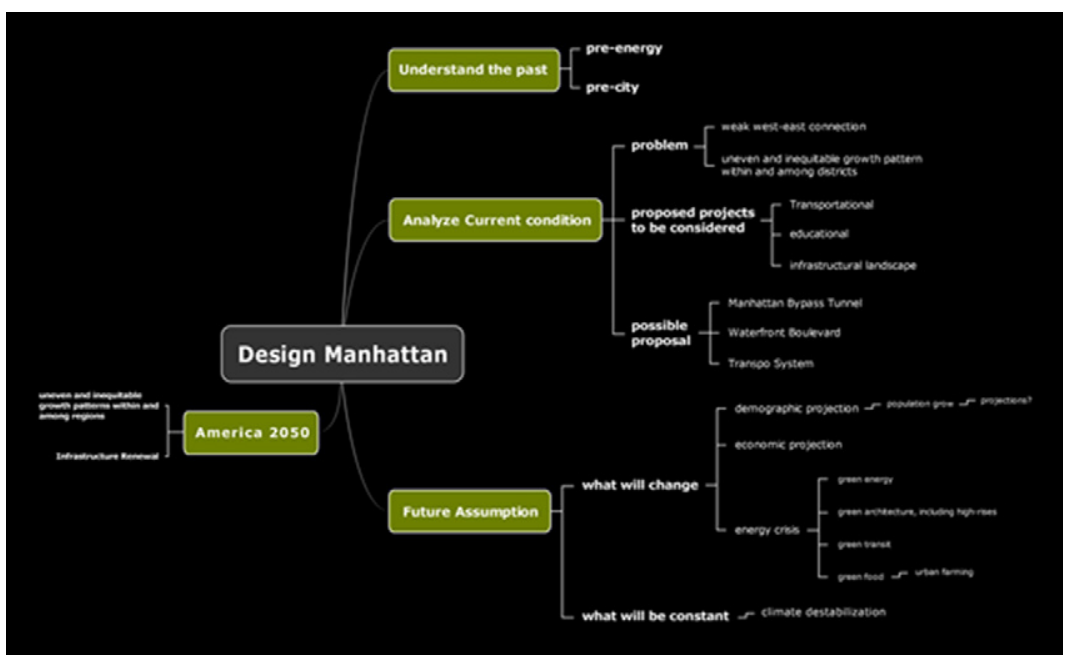

Figure 4. Urban design studio process. Not linear but fluid, enabling research, analysis and design to occur at several points during the project

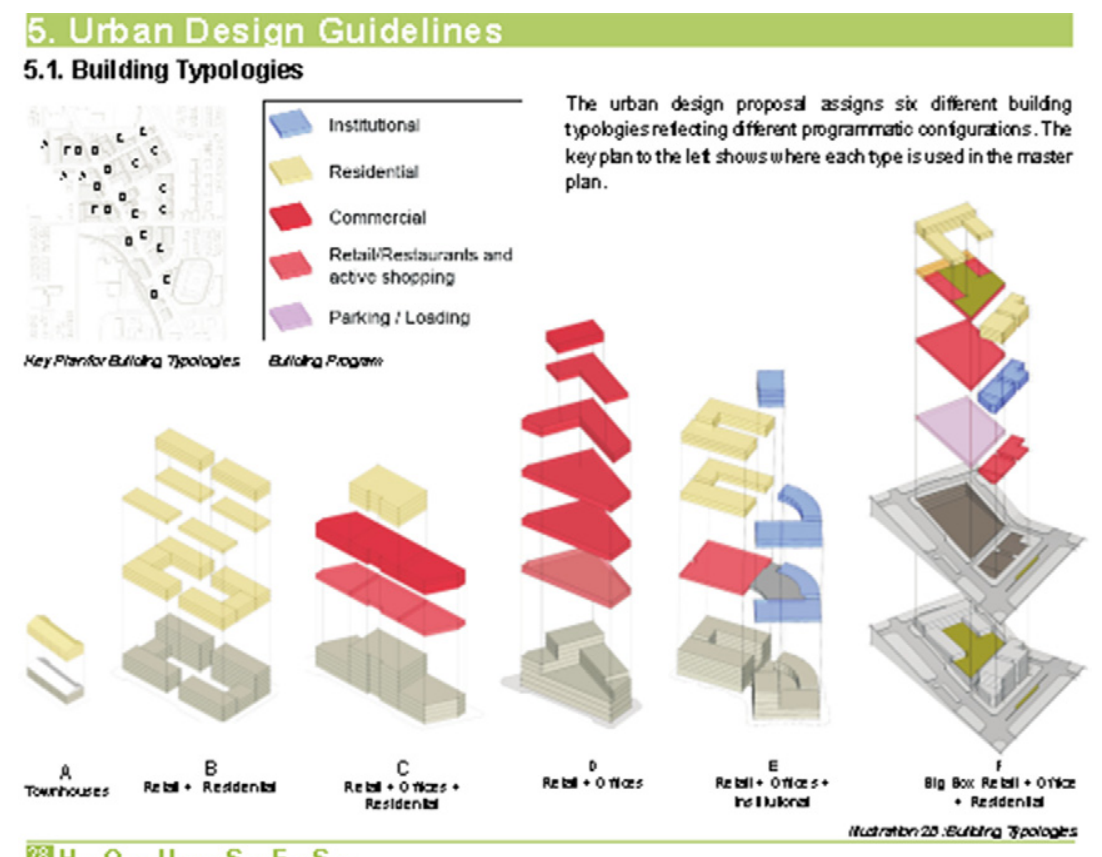

Figure 5. Studio project urban design guidelines. (Credit: Michel Banna, Alex DeCamp, Sara Hadavi, Amir Hajrasouliha and Aditya Inamdar)

Although the recommendations presented above are straightforward, putting them into effect within the short, one- to two-year time span of most graduate-level urban design programmes requires a concentrated effort. But with their application programmes can prepare students to design synthetically, communicate clearly and practice globally while reinforcing the pedagogic approaches presented in this issue of the journal of Urban Design and Planning. 